\title{
Anti-inflammatory effects of essential oils from Chamaecyparis obtusa via the cyclooxygenase-2 pathway in rats
}

\author{
BEUM-SOO AN ${ }^{1,2^{*}}$, JI-HOUN KANG ${ }^{3 *}$, HYUN YANG $^{1}$, EUI-MAN JUNG ${ }^{1}$, HONG-SEOK KANG ${ }^{1}$, \\ IN-GYU CHOI ${ }^{4}$, MI-JIN PARK ${ }^{5}$ and EUI-BAE JEUNG ${ }^{1}$ \\ ${ }^{1}$ Laboratory of Veterinary Biochemistry and Molecular Biology, College of Veterinary Medicine, \\ Chungbuk National University, Cheongju, Chungbuk 361-763; ${ }^{2}$ Department of Biomaterials Science, \\ College of National Resources and Life Science, Pusan National University, Miryang, Gyeongsangnam-do 627-706; \\ ${ }^{3}$ Laboratory of Veterinary Internal Medicine, College of Veterinary Medicine, Chungbuk National University, Cheongju, \\ Chungbuk 361-763; ${ }^{4}$ Department of Forest Sciences, College of Agriculture and Life Sciences, Seoul National University, \\ Seoul 151-921; ${ }^{5}$ Department of Forest Products, Division of Wood Chemistry and Microbiology,
} Korea Forest Research Institute, Seoul 130-712, Republic of Korea

Received December 4, 2012; Accepted April 23, 2013

DOI: $10.3892 / \mathrm{mmr} .2013 .1459$

\begin{abstract}
Essential oils are concentrated hydrophobic liquids containing volatile aromatic compounds from plants. In the present study, the essential oil of Chamaecyparis obtusa (C. obtusa), which is commercially used in soap, toothpaste and cosmetics, was extracted. Essential oil extracted from C. obtusa contains several types of terpenes, which have been shown to have anti-oxidative and anti-inflammatory effects. In the present study, we examined the anti-inflammatory effects of C.obtusa essential oil in vivo and in vitro following the induction of inflammation by lipopolysaccharides (LPS) in rats. While LPS induced an inflammatory response through the production of prostaglandin $\mathrm{E}_{2}\left(\mathrm{PGE}_{2}\right)$ in the blood and peripheral blood mononuclear cells (PMNCs), these levels were reduced when essential oil was pre-administered. Additionally, the mechanism of action underlying the anti-inflammatory effects of C. obtusa essential oil was investigated by measuring the mRNA expression of inflammation-associated genes. LPS treatment significantly induced the expression of transforming growth factor $\alpha(\mathrm{TNF} \alpha)$ and
\end{abstract}

Correspondence to: Professor Eui-Bae Jeung, Laboratory of Veterinary Biochemistry and Molecular Biology, Room 214, College of Veterinary Medicine, Chungbuk National University, 48 Gaesin-dong, Cheongju, Chungbuk 361-763, Republic of Korea E-mail: ebjeung@chungbuk.ac.kr

*Contributed equally

Abbreviations: C. obtusa, Chamaecyparis obtusa; PGE2, prostaglandin $\mathrm{E}_{2}$; $\mathrm{TNF} \alpha$, transforming growth factor $\alpha$; COX-2, cyclooxygenase-2; NSAIDS, nonsteroidal anti-inflammatory drugs; LPS, endotoxin lipopolysaccharides

Key words: Chamaecyparis obtusa, essential oil, anti-inflammation, PGE2, TNF $\alpha$, COX-2 cyclooxygenase-2 (COX-2) in rats, while $C$. obtusa essential oil inhibited this effect. Taken together, our results demonstrate that $C$. obtusa essential oil exerts anti-inflammatory effects by regulating the production of $\mathrm{PGE}_{2}$ and $\mathrm{TNF} \alpha$ gene expression through the COX-2 pathway. These findings suggest that $C$. obtusa essential oil may constitute a novel source of anti-inflammatory drugs.

\section{Introduction}

Inflammation is the most common cause of clinical pain resulting from tissue injury. The primary function of pain is to protect the organism from potential tissue-damaging stimuli. The aim of drug treatment for inflammation is to normalize pain sensitivity; however, drugs that are currently available are associated with critical side-effects and low efficacy. Non-steroidal anti-inflammatory drugs (NSAIDs) have been shown to be effective in the treatment of various disorders with inflammation and have fewer side-effects compared with steroids (1). NSAIDs function through binding to the cyclooxygenase (COX) enzymes to inhibit the production of prostaglandins from arachidonic acid. Two isoforms of the COX protein, COX-1 and -2, exist; COX-1 is constitutively expressed in the majority of organs and cells, whereas COX-2 is generally absent and is induced upon stimulation by inflammatory stimuli, including endotoxin lipopolysaccharides (LPS). This suggests that COX-2 is an inflammation-specific isoform (1-3). NSAIDs and COX-2 selective inhibitors are currently used to treat several inflammatory disorders (4-6). However, selective inhibitors of COX-2 have been associated with a risk of myocardial infarction and stroke. The recent withdrawal of conventional NSAIDs and selective COX-2 inhibitors due to their adverse cardiovascular and gastric side-effects has led to the development of alternative anti-inflammatory agents (7-9). Therefore, plant-derived natural agents, such as essential oil, are considered to be useful sources for the next generation of anti-inflammatory drugs. 
Essential oils are concentrated hydrophobic liquids containing volatile aromatic compounds from plants. They are extracted by distillation, expression or solvent extracts, and are used in perfumes, cosmetics, soaps and other products, for adding scents to incense and household products and for flavoring food and drink. Chamaecyparis obtusa (C. obtusa), a tropical tree species found in Japan and the southern region of South Korea, is used for construction and furniture due to its advantage in structural properties and natural scent. Its essential oil is extracted from pruned leaves and twigs of the C. obtusa tree, and has been commercially used in soap, toothpaste and cosmetics as a functional additive. The C. obtusa essential oil contains several types of terpenes that have been shown to exert anti-oxidative and anti-inflammatory effects, including sabinene, limonene, bornyl acetate, borneol, $\alpha$-terpineol and elemol, while essential oils from fruits contain myrcene, $\gamma$-terpinene, $p$-cymene, borneol, $\alpha$-terpineol and $\beta$-caryophyllene $(10,11)$.

In the present study, the anti-inflammatory property of the essential oil from C. obtusa was investigated in vivo and in vitro, and the ability of $C$.obtusa to serve as a source of novel pharmaceuticals was determined.

\section{Materials and methods}

Animals and treatment. Male Sprague-Dawley (SD) rats (9 weeks old) were obtained from Koatech Co., Ltd. (Pyeongtaek-si, Korea). All the animals were housed in polycarbonate cages and acclimated in an environmentally controlled room $\left(23 \pm 2^{\circ} \mathrm{C}\right.$; relative humidity, $50 \pm 10 \%$; frequent ventilation; and a 12-h light/dark cycle) prior to use as previously described (12). C. obtusa essential oil used in this study was provided by Enbita Co., Ltd. (Seoul, Korea) and was produced through steam distillation of pruned twigs and leaves of the C. obtusa tree, according to previously described methods (13). The rats were treated with $C$. obtusa essential oil to investigate its effects. A total of $50 \mathrm{ml}$ tap water or essential oil solution $(25,50$ or $100 \%)$ was administered to the rats for 2 weeks. Rats in the control group were treated with a saline solution $(0.9 \% \mathrm{NaCl})$, and the remaining rats (treatment group) were treated with $0,25,50$ or $100 \%$ solutions of $C$. obtusa essential oil in the presence or absence of LPS (Sigma-Aldrich, St. Louis, MO, USA). Rats in the LPS-treated group (LPS-positive group) were administered $1 \mu \mathrm{g} / \mathrm{kg}$ LPS via an intraperitoneal (i.p.) injection $8 \mathrm{~h}$ prior to sacrifice; rats in the control group for LPS (LPS-negative group) were injected with an equivalent volume of saline solution at the same time-point with LPS. All the experimental procedures were approved by the Ethics Committee of the Chungbuk National University (Cheongju, Korea).

Peripheral blood mononuclear cell (PMNC) isolation. PMNCs were isolated from rat peripheral blood vessels using a double density gradient centrifugation method as previously described $(14,15)$. Briefly, heparinized blood samples were overlaid on Histopaque-1077 solution (specific gravity, 1.077; Sigma-Aldrich) and Histopaque-1119 solution (specific gravity, 1.199; Sigma-Aldrich), centrifuged for $40 \mathrm{~min}$ at $700 \mathrm{x} \mathrm{g}$ and washed three times with cold phosphate-buffered saline (PBS). The viability of PMNCs, determined by trypan blue dye exclusion, was $>97 \%$. The PMNCs were resuspended in RPMI-1640 medium (Sigma-Aldrich) supplemented with $2 \mathrm{mM}$ L-glutamine, $0.02 \mathrm{mg} / \mathrm{ml}$ gentamicin and $5 \%$ heat-inactivated fetal bovine serum (FBS; Invitrogen, Grand Island, NY, USA) for use in subsequent experiments.

Prostaglandin $E_{2}\left(P G E_{2}\right)$ ELISA. For the in vivo experiment, rats were treated with various solutions of $C$.obtusa essential oil in the presence or absence of LPS. Following treatment, serum was collected from peripheral blood vessels and subjected to a $\mathrm{PGE}_{2}$ ELISA assay. For the in vitro assay, rats were treated with the essential oil solutions for 2 weeks. PMNCs were harvested from rat peripheral blood vessels and plated on 24-well plates at a density of $0.5 \times 10^{5}$. The PMNCs were then stimulated with $10 \mathrm{ng} / \mathrm{ml}$ LPS for the indicated times, and the supernatant was collected. For the $\mathrm{PGE}_{2}$ ELISA assay, the collected serum and supernatant were analyzed using a monoclonal $\mathrm{PGE}_{2}$ ELISA kit (Cayman Chemical, Ann Arbor, MI, USA).

Quantification of mRNA by RT-PCR. Total cellular RNA was extracted using TRIzol reagent (Invitrogen), and the concentration of RNA was determined at $260 \mathrm{~nm}$. RT-PCR was performed as previously described (13). Briefly, total RNA $(1 \mu \mathrm{g})$ was reverse transcribed into complementary DNA (cDNA) using M-MLV reverse transcriptase (Invitrogen) and a random primer (9-mer; Takara Bio, Inc., Shiga, Japan). The cDNA $(1 \mu 1)$ was used for PCR under standard conditions $(13,16)$; denaturation at $95^{\circ} \mathrm{C}$ for $30 \mathrm{sec}$, annealing at $55^{\circ} \mathrm{C}$ for $30 \mathrm{sec}$ and extension at $72^{\circ} \mathrm{C}$ for $1 \mathrm{~min}$. The primer sequences used were as follows: COX-2 sense, 5'-TACCCGGACTGGATTCTACG-3' and antisense, 5'-AAGTTGGTGGGCTGTCAATC-3'; TNF $\alpha$ sense, 5'-CTGAGCTCAAGCCCTGGTAT-3' and antisense, 5' - GGTCAGAGTA A TGGGGGTCA-3'; and cytochrome $c$ oxidase subunit 1 (1A) sense, 5'-CCAGGGTTTGGAATTATTTC-3' and antisense, 5'-GAAGATAAACC CTAAGGCTC-3', which was used as an internal control. The PCR products $(10 \mu \mathrm{l})$ were separated on $2 \%$ agarose gel and stained with ethidium bromide (EtBr). The gel image was captured and analyzed using Quantity One software (Gel Doc EQ; Bio-Rad, Hercules, CA, USA).

Statistical analysis. Data were analyzed by non-parametric one-way analysis of variance using the Kruskal-Wallis test, followed by Dunnett's test for multiple comparisons. Data values were converted to ranks for these tests. All the statistical analyses were performed with SPSS for Windows (SPSS, Inc., Chicago, IL, USA). $\mathrm{P}<0.05$ was considered to indicate a statistically significant difference.

\section{Results}

Effect of essential oil from C. obtusa on serum $P G E_{2}$ concentration. The regulation of serum $\mathrm{PGE}_{2}$ by essential oils from C. obtusa was investigated. Rats were treated with various concentrations of the essential oil for 2 weeks and $1 \mu \mathrm{g} / \mathrm{kg}$ LPS was injected to induce an inflammatory reaction following treatment with the essential oil.

To analyze the levels of serum $\mathrm{PGE}_{2}$, ELISA with a specific antibody for $\mathrm{PGE}_{2}$ was performed. The levels of LPS-induced 


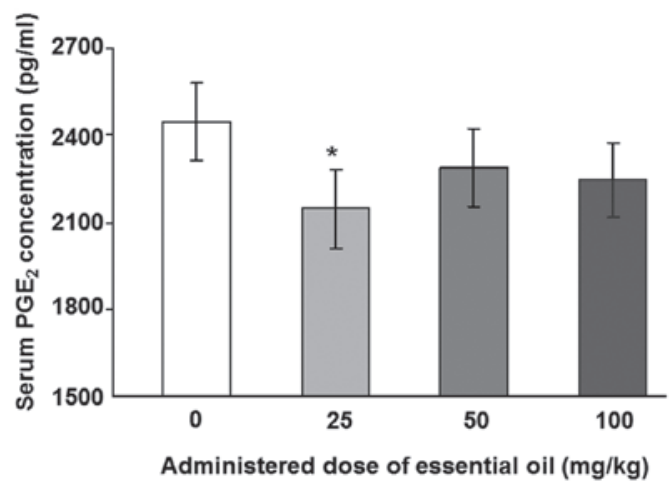

Figure 1. Effects of various doses of Chamaecyparis obtusa essential oil on lipopolysaccharide (LPS)-induced concentrations of prostaglandin $\mathrm{E}_{2}$ $\left(\mathrm{PGE}_{2}\right)$ in the serum of rats. The rats were treated via oral administration of the essential oils for 2 weeks, and $1 \mu \mathrm{g} / \mathrm{kg}$ LPS was intraperitoneally injected on the last day of treatment. Serum samples were collected from rat blood samples $8 \mathrm{~h}$ after the LPS injection. The serum samples were filtered with a $0.45-\mu \mathrm{m}$ pore size membrane and $\mathrm{PGE}_{2}$ concentrations were determined using ELISA. " $\mathrm{P}=0.036$ compared with control group, as determined by oneway ANOVA followed by a Dunnett's test $(n=4)$.

$\mathbf{A}$

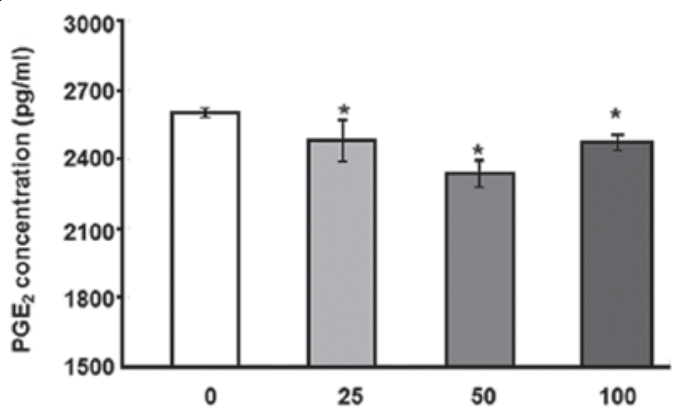

Administered dose of essential oil (mg/kg)

B

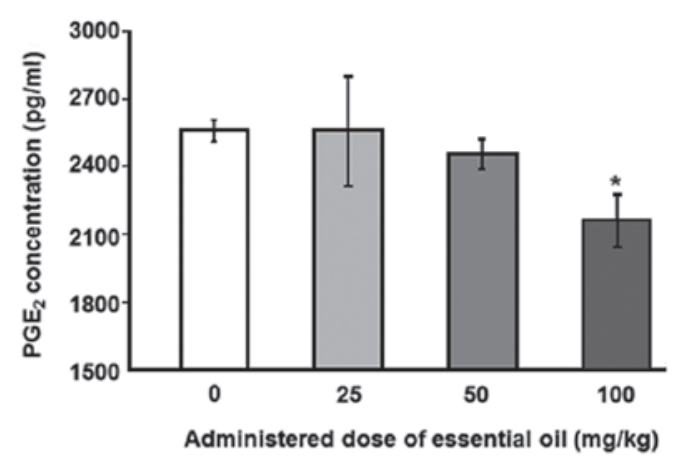

Figure 2. Effect of various doses of Chamaecyparis obtusa essential oil on lipopolysaccharide (LPS)-induced production of prostaglandin $\mathrm{E}_{2}\left(\mathrm{PGE}_{2}\right)$ using peripheral blood mononuclear cells (PMNCs). PMNCs were isolated from blood samples obtained from rats treated via oral administration of the essential oil for 2 weeks. Isolated PMNCs were stimulated by $10 \mathrm{ng} / \mathrm{ml}$ LPS for (A) 12 or (B) $24 \mathrm{~h}$, and the culture supernatants were harvested and filtered with a $0.45-\mu \mathrm{m}$ pore size membrane. $\mathrm{PGE}_{2}$ concentrations in the culture supernatant were determined using ELISA. "P $\leq 0.001$ compared with control group, as determined by one-way ANOVA followed by a Dunnett's test $(n=4)$.

serum $\mathrm{PGE}_{2}$ were significantly reduced in animals treated with essential oil at a dose of $25 \mathrm{mg} / \mathrm{kg}$ (Fig. 1). Other concentrations of C. obtusa essential oil (50 and $100 \mathrm{mg} / \mathrm{kg}$ ) also
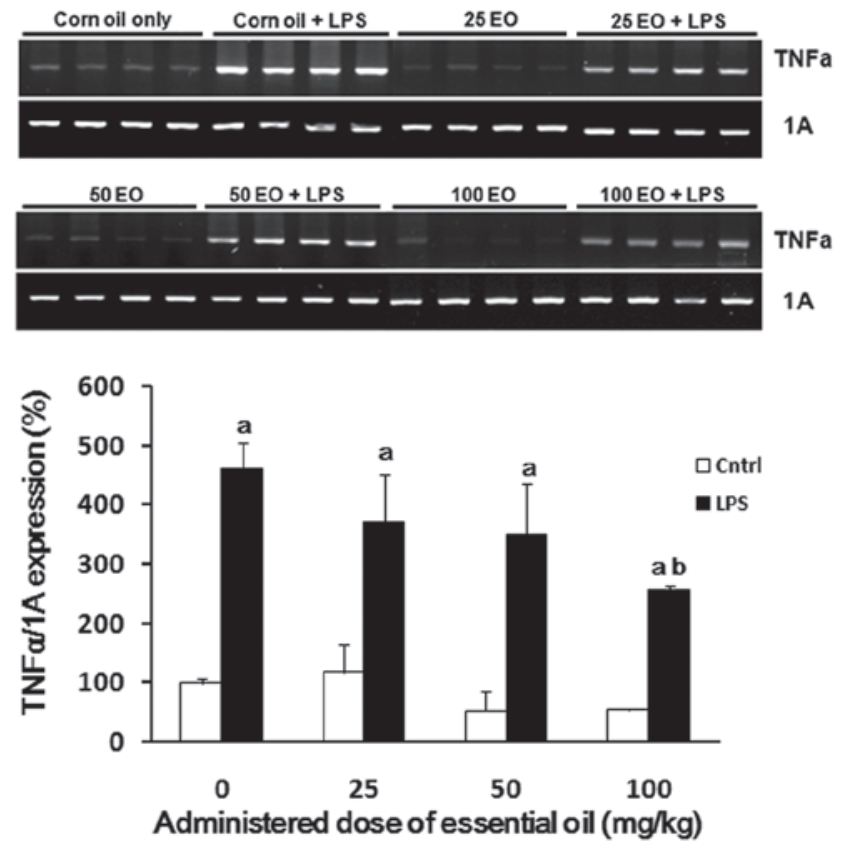

Figure 3. Effect of Chamaecyparis obtusa essential oil (EO) on lipopolysaccharide (LPS)-induced expression of tumor necrosis factor $\alpha$ (TNF $\alpha)$ mRNA. The rats were treated via oral administration of various doses of the essential oil for 2 weeks, and $1 \mu \mathrm{g} / \mathrm{kg}$ LPS was intraperitoneally injected on the last day of treatment. The mRNA samples were extracted from the lungs of rats $8 \mathrm{~h}$ after the LPS injection and analyzed by RT-PCR with primers for TNF $\alpha$ as described in Material and methods. ${ }^{\mathrm{a}} \mathrm{P}<0.05$ compared with the LPS-negative group; ${ }^{b} \mathrm{P}<0.05$ compared with the LPS-positive control group.

reduced the levels of serum $\mathrm{PGE}_{2}$; however, these results were not statistically significant. Next, the effects of essential oil were evaluated, using PMNCs to verify the increase in serum $\mathrm{PGE}_{2}$ in the rats. PMNCs were isolated following treatment with various concentrations of the essential oil for 2 weeks and administration of $10 \mathrm{ng} / \mathrm{ml}$ LPS for 12 (Fig. 2A) or $24 \mathrm{~h}$ (Fig. 2B). The essential oil was shown to have protective effects on LPS-induced inflammation by reducing $\mathrm{PGE}_{2}$ secretion in the PMNCs. The 12-h LPS-induced $\mathrm{PGE}_{2}$ secretion level was inhibited by treatment with the essential oil at all the doses examined, and the maximum inhibition was observed at a dose of $50 \mathrm{mg} / \mathrm{kg}$. The inhibition of $\mathrm{PGE}_{2}$ production induced by LPS treatment for $24 \mathrm{~h}$ was shown to be significant only when $100 \mathrm{mg} / \mathrm{kg}$ of the essential oil was applied.

Effect of essential oils from C. obtusa on expression of the inflammation-related genes $T N F \alpha$ and $C O X-2$ in lung tissue. To examine the possible mechanisms of action underlying the anti-inflammatory effects of the essential oil, we analyzed the expression of inflammation-associated genes in the lungs of rats following treatment with the essential oil in the presence or absence of LPS. As expected, LPS enhanced the expression of TNF $\alpha$ (Fig. 3). LPS-induced TNF $\alpha$ expression in the lung was reduced by administration of the essential oil in a dose-dependent manner, where the most potent effect was observed at a dose of $100 \mathrm{mg} / \mathrm{kg}$ (56\% reduction).

COX-2 mRNA expression was also assessed to determine the mechanism via which the essential oil exerts it anti-inflam- 

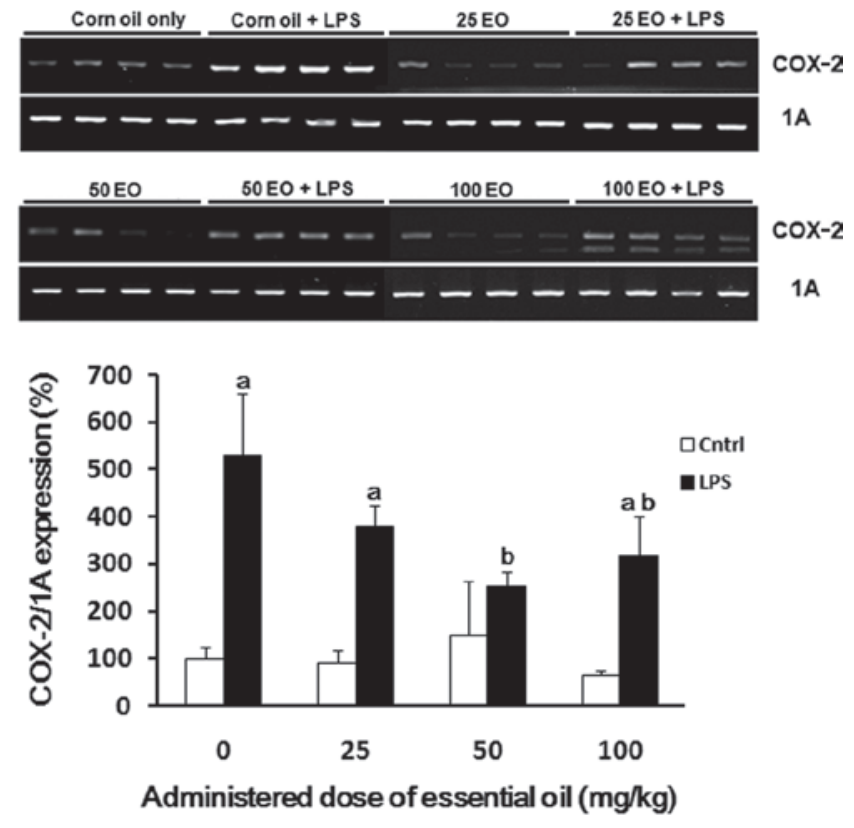

Figure 4. Effect of Chamaecyparis obtusa essential oil (EO) on lipopolysaccharide (LPS)-induced expression of cyclooxygenase-2 (COX-2) mRNA. The rats were treated via oral administration of various doses of the essential oil for 2 weeks, and $1 \mu \mathrm{g} / \mathrm{kg}$ LPS was intraperitoneally injected on the last day of treatment. The mRNA samples were extracted from the lungs of rats $8 \mathrm{~h}$ after the LPS injection, and COX-2 expression was analyzed by RT-PCR. ${ }^{\text {a }} \mathrm{P}<0.05$ compared with the LPS-negative group; ${ }^{\text {b }} \mathrm{P}<0.05$ compared with the LPS-positive control group.

matory effects (Fig. 4). The expression of COX-2 was increased by LPS treatment (up to 5-fold) compared with the control group (Fig. 4). The induction of COX-2 expression by LPS treatment was inhibited by pre-administration of the essential oil; this inhibition was most marked at a dose of $50 \mathrm{mg} / \mathrm{kg}$.

\section{Discussion}

Since ancient times, plants have been used for their medicinal properties, which are partially attributed to essential oils (17). Essential oils are natural, complex and multi-component oils composed mainly of terpenes with additional components (18). Essential oils are widely used to prevent and treat human diseases, including cancer and cardiovascular diseases. Furthermore, their bioactivity as antibacterial, antiviral, antioxidant and antidiabetic agents has previously been reported (18). Recent studies have demonstrated the anti-inflammatory properties of essential oils from several plants (19-21). In the present study, we demonstrated that the essential oil from $C$. obtusa has an anti-inflammatory effect on LPS-induced inflammation in rats. The serum concentration of $\mathrm{PGE}_{2}$ was reduced when the rats were treated with the essential oil. In addition, the synthesis of $\mathrm{PGE}_{2}$ from blood PMNCs showed concomitant results with that of serum. These results suggest that $\mathrm{PGE}_{2}$ is synthesized by blood monocytes following stimulation by LPS, and that this inflammatory process is ameliorated by $C$. obtusa essential oil.

$\mathrm{PGE}_{2}$ is produced in the body and accumulates with LPS treatment through stimulation of the COX pathway. The quantitative analysis of $\mathrm{PGE}_{2}$ represents inflammatory activities in vivo and in vitro (22). In the present study, LPS-induced $\mathrm{PGE}_{2}$ production was reduced in the serum and PMNCs of rats by treatment with $C$. obtusa essential oil. Similar results have been previously reported; Hyptis pectinata essential oil has been shown to have anti-inflammatory effects by inhibiting the production of $\mathrm{PGE}_{2}$ and nitric oxide (23). Cryptomeria japonica essential oil has been demonstrated to inhibit the LPS-induced production of $\mathrm{PGE}_{2}$ and cytokines in macrophages, including interleukin (IL)-1 $\beta$ and IL-6 (24); furthermore, the chemical composition of Cryptomeria japonica essential oil was analyzed and its major components were revealed to be elemol and sabinene. Elemol and sabinene are also known to be terpenes of $C$.obtusa essential oil $(10,11)$.

In the present study, we investigated the effects of $C$.obtusa essential oil on the regulation of $\mathrm{TNF} \alpha \mathrm{mRNA}$ expression following LPS treatment. As expected, the expression of $\mathrm{TNF} \alpha$ was increased in the lung tissues of rats following LPS treatment, which implied induction of the immune response by LPS. Furthermore, the induced expression of TNF $\alpha$ was abrogated by treatment with $C$. obtusa essential oil. To verify the mechanism of action underlying the anti-inflammatory effects of $C$. obtusa essential oil, we also examined the regulation of COX-2 expression levels. Pathogen-associated immune responses are antigenic, and are able to activate monocytes or macrophages to synthesize various inflammatory cytokines, including IL-1, IL-6 and TNF, which have been reported to be mediated via COX expression (24-26). The expression of COX-2 was increased following treatment with LPS, and this upregulation was inhibited by $C$. obtusa essential oil in a dose-dependent manner. These results suggest that the anti-inflammatory effects of this essential oil occur via COX-2-mediated regulation of PGE2 and TNF $\alpha$ signaling pathways.

The anti-inflammatory effects of essential oils through the COX-2 signaling pathway have been previously demonstrated; the essential oil from Houttuynia cordata has been shown to possess anti-inflammatory properties through inhibiting the LPS-induced production of $\mathrm{PGE}_{2}$ (9). In this study, the anti-inflammatory effects of the essential oil were mediated by the inhibition of COX-2 gene expression, while COX-1, the non-inflammation-specific isoform, was not involved. Katsuawa et al (27) also demonstrated that the essential oil of lemongrass suppresses COX-2 promoter activity in human macrophage-like U937 cells.

In conclusion, our results demonstrate that $C$. obtusa essential oil exerts it anti-inflammatory effects by regulating the production of $\mathrm{PGE}_{2}$ in the blood and the gene expression of TNF $\alpha$ in rats. The anti-inflammatory effects of C. obtusa essential oil are mediated by inhibiting the expression of the inflammation-specific COX-2 enzyme. Our results suggest that $C$. obtusa essential oil is a novel source of inflammation-specific pharmacological drugs. However, further studies are required to investigate the specific mechanisms of action and potential side-effects of C. obtusa essential oil.

\section{Acknowledgements}

This study was supported by a National Research Foundation of Korea (NRF) grant funded by the Korea government (MEST; no. 2010-0011433). 


\section{References}

1. Simmons DL, Botting RM and Hla T: Cyclooxygenase isozymes: the biology of prostaglandin synthesis and inhibition. Pharmacol Rev 56: 387-437, 2004

2. Smith WL: Nutritionally essential fatty acids and biologically indispensable cyclooxygenases. Trends Biochem Sci 33: 27-37, 2008

3. Koki A, Khan NK, Woerner BM, et al: Cyclooxygenase-2 in human pathological disease. Adv Exp Med Biol 507: 177-184, 2002.

4. Zha S, Yegnasubramanian V, Nelson WG, Isaacs WB and De Marzo AM: Cyclooxygenases in cancer: progress and perspective. Cancer Lett 215: 1-20, 2004.

5. Cuzick J, Otto F, Baron JA, et al: Aspirin and non-steroidal anti-inflammatory drugs for cancer prevention: an international consensus statement. Lancet Oncol 10: 501-507, 2009.

6. Thun MJ and Blackard B: Pharmacologic effects of NSAIDs and implications for the risks and benefits of long-term prophylactic use of aspirin to prevent cancer. Recent Results Cancer Res 181: 215-221, 2009.

7. Mukherjee D: Selective cyclooxygenase-2 (COX-2) inhibitors and potential risk of cardiovascular events. Biochem Pharmacol 63 817-821, 2002.

8. Coruzzi G, Venturi N and Spaggiari S: Gastrointestinal safety of novel nonsteroidal antiinflammatory drugs: selective COX-2 inhibitors and beyond. Acta Biomed 78: 96-110, 2007.

9. Li W, Zhou P, Zhang Y and He L: Houttuynia cordata, a novel and selective COX-2 inhibitor with anti-inflammatory activity. J Ethnopharmacol 133: 922-927, 2011

10. Joo SS, Yoo YM, Ko SH, et al: Effects of essential oil from Chamaecypris obtusa on the development of atopic dermatitislike skin lesions and the suppression of Th cytokines. J Dermatol Sci 60: 122-125, 2010.

11. Singh BK, Tripathi M, Chaudhari BP, Pandey PK and Kakkar P: Natural terpenes prevent mitochondrial dysfunction, oxidative stress and release of apoptotic proteins during nimesulide-hepatotoxicity in rats. PLoS One 7: e34200, 2012.

12. Lee GS, Byun HS, Kim MH, et al: The beneficial effect of the sap of Acer mono in an animal with low-calcium diet-induced osteoporosis-like symptoms. Br J Nutr 100: 1011-1018, 2008.

13. Lee GS, Hong EJ, Gwak KS, et al: The essential oils of Chamaecyparis obtusa promote hair growth through the induction of vascular endothelial growth factor gene. Fitoterapia 81: 17-24, 2010.

14. Lee SC, Ju SA, Pack HN, et al: 4-1BB (CD137) is required for rapid clearance of Listeria monocytogenes infection. Infect Immun 73: 5144-5151, 2005.
15. Hasegawa H, Suzuki K, Nakaji S and Sugawara K: Analysis and assessment of the capacity of neutrophils to produce reactive oxygen species in a 96-well microplate format using lucigenin- and luminoldependent chemiluminescence. J Immunol Methods 210: 1-10, 1997.

16. Vo TT, An BS, Yang H, Jung EM, Hwang I and Jeung EB: Calbindin-D9k as a sensitive molecular biomarker for evaluating the synergistic impact of estrogenic chemicals on GH3 rat pituitary cells. Int J Mol Med 30: 1233-1240, 2012.

17. Takayama C, de-Faria FM, de Almeida AC, et al: Gastroprotective and ulcer healing effects of essential oil from Hyptis spicigera Lam. (Lamiaceae). J Ethnopharmacol 135: 147-155, 2011.

18. Edris AE: Pharmaceutical and therapeutic potentials of essential oils and their individual volatile constituents: a review. Phytother Res 21: 308-323, 2007.

19. Silva FV, Guimarães AG, Silva ER, et al: Anti-inflammatory and anti-ulcer activities of carvacrol, a monoterpene present in the essential oil of oregano. J Med Food 15: 984-991, 2012.

20. Lima DK, Ballico LJ, Rocha Lapa F, et al: Evaluation of the antinociceptive, anti-inflammatory and gastric antiulcer activities of the essential oil from Piper aleyreanum C.DC in rodents. J Ethnopharmacol 142: 274-282, 2012.

21. Veras HN, Araruna MK, Costa JG, et al: Topical antiinflammatory activity of essential oil of Lippia sidoides Cham: possible mechanism of action. Phytother Res 27: 179-185, 2012.

22. Hennebert O, Pelissier MA, Le Mee S, Wülfert E and Morfin R: Anti-inflammatory effects and changes in prostaglandin patterns induced by 7 beta-hydroxy-epiandrosterone in rats with colitis. J Steroid Biochem Mol Biol 110: 255-262, 2008.

23. Raymundo LJ, Guilhon CC, Alviano DS, et al: Characterisation of the anti-inflammatory and antinociceptive activities of the Hyptis pectinata (L.) Poit essential oil. J Ethnopharmacol 134: 725-732, 2011.

24. Yoon WJ, Kim SS, Oh TH, Lee NH and Hyun CG: Cryptomeria japonica essential oil inhibits the growth of drug-resistant skin pathogens and LPS-induced nitric oxide and pro-inflammatory cytokine production. Pol J Microbiol 58: 61-68, 2009.

25. Chao LK, Hua KF, Hsu HY, et al: Cinnamaldehyde inhibits pro-inflammatory cytokines secretion from monocytes/macrophages through suppression of intracellular signaling. Food Chem Toxicol 46: 220-231, 2008

26. Takeda K, Kaisho T and Akira S: Toll-like receptors. Annu Rev Immunol 21: 335-376, 2003.

27. Katsukawa M, Nakata R, Takizawa Y, Hori K, Takahashi S and Inoue H: Citral, a component of lemongrass oil, activates PPARalpha and gamma and suppresses COX-2 expression. Biochim Biophys Acta 1801: 1214-1220, 2010. 